https://helda.helsinki.fi

Three-dimensional printed surgical templates for fresh cadaveric osteochondral allograft surgery with dimension verification by multivariate computed tomography analysis

\author{
Huotilainen, Eero
}

2019-08

Huotilainen , E , Salmi , M \& Lindahl , J 2019 , ' Three-dimensional printed surgical templates for fresh cadaveric osteochondral allograft surgery with dimension verification by multivariate computed tomography analysis ' , Knee , vol. 26 , no. 4 , pp. 923-932 . https://doi.org/10.1016/j.knee.201

http://hdl.handle.net/10138/320948

https://doi.org/10.1016/j.knee.2019.05.007

publishedVersion

Downloaded from Helda, University of Helsinki institutional repository.

This is an electronic reprint of the original article.

This reprint may differ from the original in pagination and typographic detail.

Please cite the original version. 


\title{
Three-dimensional printed surgical templates for fresh cadaveric osteochondral allograft surgery with dimension verification by multivariate computed tomography analysis
}

\author{
Eero Huotilainen ${ }^{\mathrm{a}, *}$, Mika Salmi ${ }^{\mathrm{a}}$, Jan Lindahl ${ }^{\mathrm{b}}$ \\ a School of Engineering, Aalto University, Espoo, Finland \\ ${ }^{\mathrm{b}}$ Helsinki University Central Hospital, Helsinki and Uusimaa Hospital District, Helsinki, Finland
}

\section{A R T I C L E I N F O}

Article history:

Received 29 December 2017

Received in revised form 19 April 2019

Accepted 9 May 2019

\section{Keywords:}

Fresh cadaveric osteochondral surgery

$3 \mathrm{D}$ printing

Computer-assisted surgery

\begin{abstract}
A B S T R A C T
Background: The fit of the allograft is a particular concern in fresh cadaveric osteochondral allograft (FOCA) surgery. Digital design and fabrication were utilized in conjunction with traditional surgery to enable efficient discovery and reproduction of appropriately dimensioned allograft.

Methods: A patient with large osteochondral defects in the lateral femoral condyle was to undergo FOCA surgery. A digital virtual operation was performed, based on computed tomography $(\mathrm{CT})$ images of the patient. Polyamide saw templates were manufactured using a selective laser sintering process, and gypsum powder was used to manufacture preoperative and intraoperative medical models with binder jetting process. The design dimensions were verified numerically by determining the intactness of the section surface and allograft volume based on four independent measurements of the initial design, and an automated design optimization strategy was postulated. For the surgery, a lateral longitudinal approach was employed.

Results: The virtual operation allowed an efficient design of the saw templates. Their shape and dimensions were verified with a numerical CT analysis method. The allograft dimensions (medial-lateral/superior-inferior/anterior-posterior) were approximately 40/28.5/24 mm, respectively, with the anterosuperior corner diagonally removed, yielding a section volume of approximately $16.5 \mathrm{~cm}^{3}$. These manually chosen dimensions were reminiscent of the corresponding computationally optimized values.

Conclusions: Use of computer-aided design in virtual operation planning and threedimensional printing in the fabrication of designed templates allowed for an efficient FOCA procedure and accurate allograft fitting. The numerical optimization method allowed for a semiautomated design process, which could in turn be realized also with surgical navigation or robotic surgery methods.
\end{abstract}

(c) 2019 Elsevier B.V. All rights reserved.

\footnotetext{
* Corresponding author at: Puumiehenkuja 5 A, 02150 Espoo, Finland.

E-mail address: eero.huotilainen@aalto.fi. (E. Huotilainen).
} 


\section{Introduction}

Three-dimensional (3D) printing, formally referred to as additive manufacturing, has been increasingly used in a medical setting for the lean production of medical models, external fixators and prostheses, surgical tools, inert implants, and biomanufacturing purposes [1]. As high-end 3D printing applications develop, autotransplants and fresh cadaveric allografts may be replaced with direct printing of functioning bio-compatible tissue (i.e. bioprinting [2,3]). Currently, however, allografts are required in severe orthopaedic traumata and conditions. A substantial clinical challenge lies in the proper fitting of the graft; in order to provide adequate level of mechanical support and osseointegration, the 3D interfaces should be properly aligned. This can be achieved with careful surgical iteration, which may in turn significantly extend operation times. Hence, it is paramount to utilize the modern techniques available to maximize the operational outcome of allografts without lengthening the surgical timespan. While preoperative 3D-printed medical models are well known and a prevalent research area [4-8], personalized surgical tools for specific complex operations remain a less prevalent application. This may be because of the extensive need for cross-disciplinary communication and collaboration in the preoperative and virtual planning process, a critical process for successful results in clinical additive manufacturing procedures [9]. The short lead times required for the creation of these tools also complicate their use for time-critical surgeries.

In this study, we evaluated a cross-disciplinary process where a patient suffering from large osteochondral defects in the lateral femoral condyle was treated with fresh cadaveric osteochondral allograft (FOCA) surgery. As a proper fit of the allograft was essential for adequate osseointegration of the sectioned area, digital design and manufacture were used to assist in the operation. Based on the computed tomography (CT) images of the recipient, a virtual operation was performed simulating the planned surgery. A set of surgery- and patient-specific surgical tools (saw templates) and medical models (a preoperative and intraoperative model) were 3D printed and used in the operation. In addition, to verify the part dimensions, planned section lines were reconstructed by varying four independent measurements of the design to present their individual (along one varied variable) and joint (along two varied variables) effects on the surface intactness and section volume. Finally, a global four-variable optimization strategy was presented as a potential semi-automated design approach.

\section{Materials and methods}

\subsection{Patient and surgical plan}

A 22-year-old male patient had a symptomatic osteochondral defect in the posterior part of the lateral femoral condyle due to a large osteochondritis dissecans (OCD) lesion. After a failed fixation and autologous bone transplantation the patient had worsening knee pain, and signs of early secondary osteoarthritis in the lateral tibiofemoral compartment. Due to osteochondral defect and degenerative changes in the lateral knee compartment, the mechanical axis of the leg was in slight valgus. This led to the decision to reconstruct the destructed posterior part of the condyle with FOCA. Valgus deformity was two to three degrees and

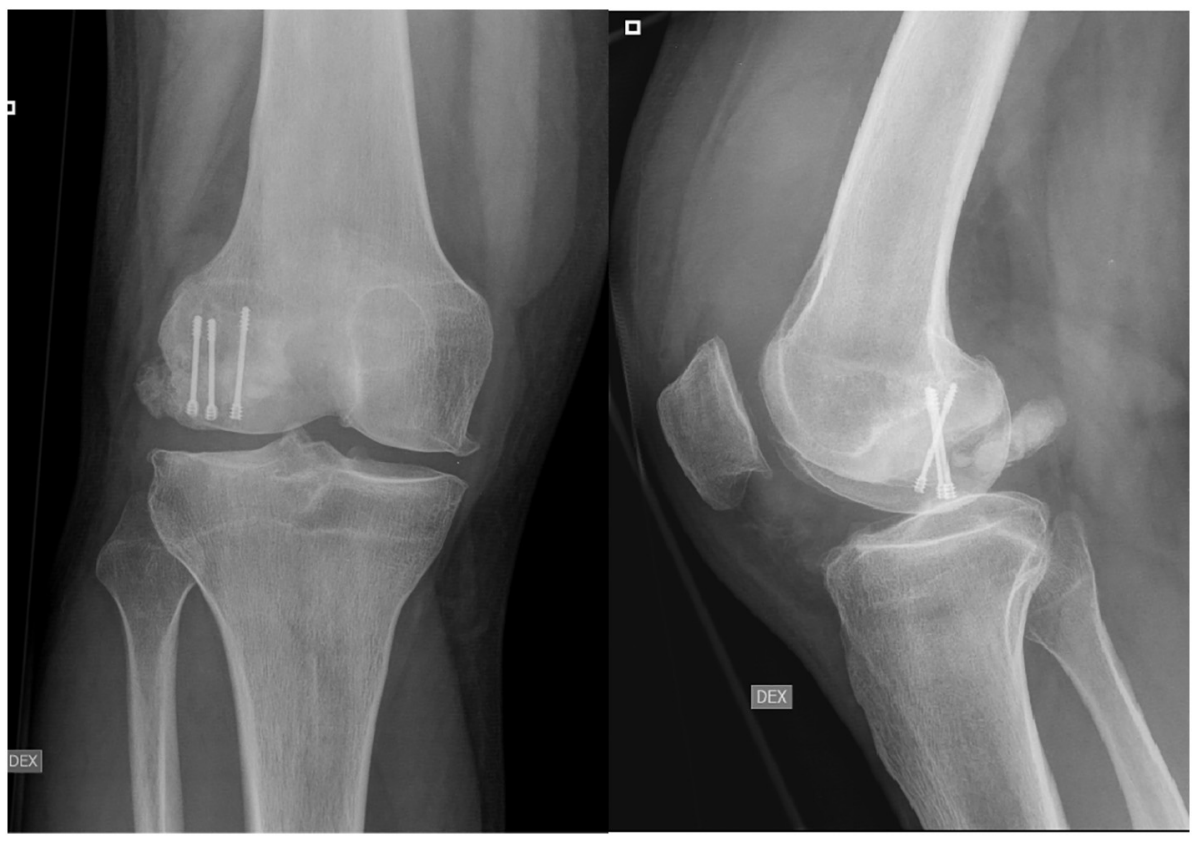

Figure 1. Preoperative radiographs. 
was not big enough to perform simultaneous distal femoral osteotomy. Preoperative radiographs of the knee are shown in Figure 1.

The surgical operation was to be performed from the lateral side of the femur. To enhance the fit of the allograft to the recipient's knee, a set of patient-specific saw templates were digitally designed and produced by 3D printing.

The responsible surgeon transferred information to the engineers responsible for the procedures described in this study via several preoperative meetings, stipulating the following requirements. (1) The defective part of the femur should be completely removed and replaced with the allograft transplant. (2) The total volume of the transplant should be minimized because increasing the amount of transplanted tissue hinders the osseointegration process. (3) The vertical and horizontal lengths of the nondiagonal vertices of the templates (see Figure 2(b)) should be sufficient to fasten the transplant. (4) The drilling from the lateral side should penetrate the entire lateral condyle; however, the drilling should not proceed into the intercondylar fossa to avoid cutting the anterior cruciate ligament.

\subsection{The virtual operation}

The right knee was imaged using a CT device (General Electric, planar resolution $0.3906 \mathrm{~mm}$, slice thickness 1.25 mm, field of view (FOV) diameter $200 \mathrm{~mm}, \mathrm{kVp}$ at 120). The resulting Digital Imaging and Communications in Medicine (DICOM) image set was opened with the OsiRiX [10] medical imaging software package (version 2.7.5) and segmented at 150 Hounsfield units.

Subsequently, the segmentation result was exported as a stereolithography (STL) file format and transferred to VisCam RP 4.0 (Marcam Engineering, Bremen, Germany) Computer-assisted design/computer-assisted manufacture (CAD/CAM) software suite for further processing. A solid block was created in VisCam so that its union with the segmented model of the defective knee would describe the part that was to be resected from the lateral femoral condyle. The shape was a heptahedron, as shown in Figure 3, with approximately a $45^{\circ}$ diagonal anterosuperior cut. The block was positioned on top of the defective area, and a Boolean subtraction was performed between the objects. This yielded a section surface candidate that was visually assessed for pores (indicating a small section), and the Boolean cutter was iteratively enlarged and translated until the section surfaces were intact. Finally, the section volume was verified not to be overestimated by decreasing the Boolean cutter dimensions, and verifying that pores started to appear beyond a safety margin of two to three millimetres.

\subsection{Design and manufacture of medical models and sawing templates}

The final Boolean cut yielded a model of the patient's knee (Figure 4, green model). This was used to produce several medical models for the surgeons. For the preoperative planning phases, a model was manufactured with a ZPrinter 450 (Z Corporation, acquired by 3D Systems, Rock Hill, SC, USA) 3DP-device from ZP 151 powder. This model was used by the surgeon for assessment, operation planning, and knowledge transfer to the engineers, by pen-marking areas of interest and special consideration. Another model was manufactured via the same 3D printing process for intraoperative use. Because the material (composite starch) used was not biocompatible, the model was placed in a sterile plastic bag.

Using the results of the virtual operation, the surface of the cutting plane was extracted and imported into 3DataExpert 7.0 (DeskArtes Oy, Espoo, Finland) CAD/CAM software. Its shape was sufficiently retained, and two volumetric meshes were extruded from the surface: a positive and negative offset. The resulting models were complementary; i.e. the larger volumetric mesh contained the dimensions of the allograft, and the smaller mesh contained the dimensions of the planned section at patient's knee (see Figure 4; the smaller (inner) template was fitted into the virtual knee model). The planar surfaces were aligned. The sawing templates were produced with a selective laser sintering process from a polyamide (PA) material.
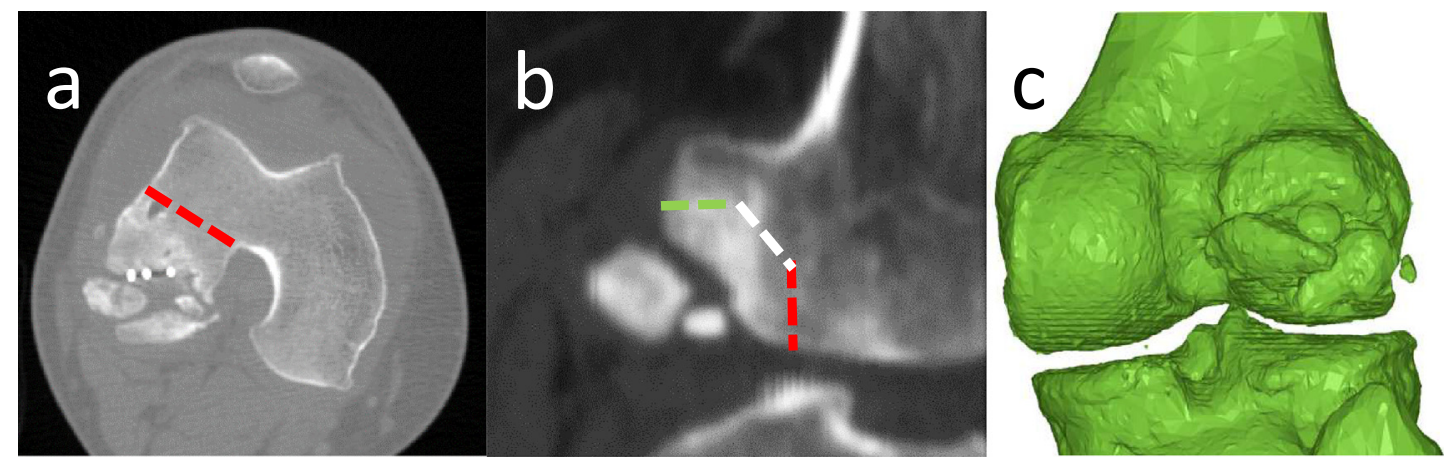

Figure 2. (a) Axial computed tomography slice showing the defective area and approximate horizontal extent of the section (red). The bright dots in the image were the fixating screws that held the defect together at that preoperative stage. (b) Sagittal view. The surgery was performed from lateral side, with a vertical cut (red), horizontal cut (green), and diagonal cut (white). (c) Reconstructed (150 HU) preoperative posterior view. 


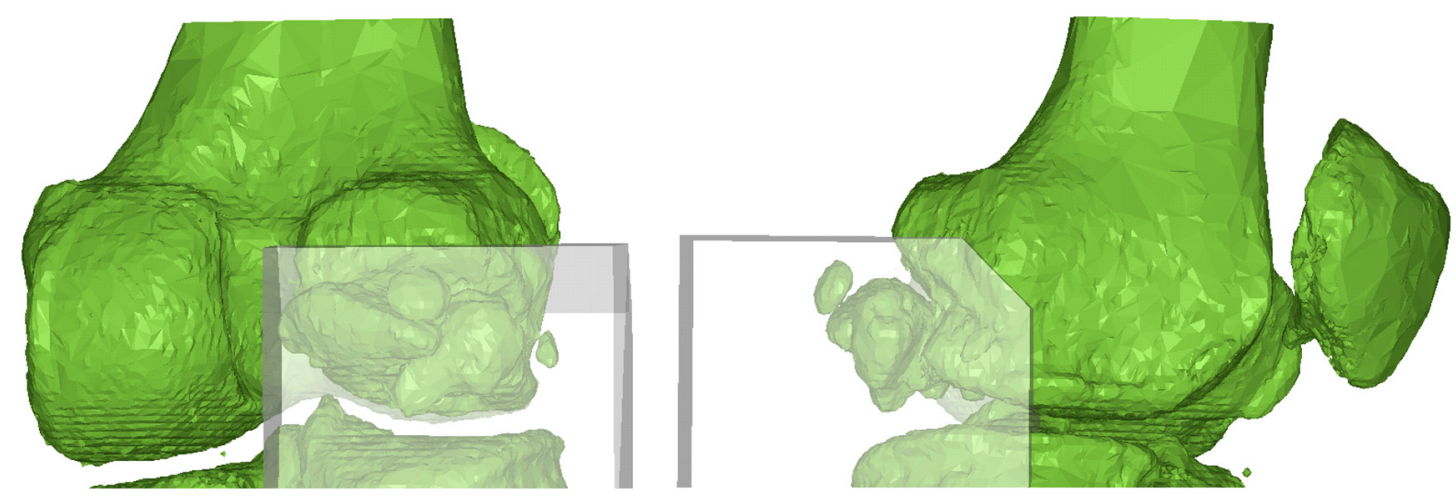

Figure 3. Boolean cutter required in the virtual operation.

\subsection{Multivariate CT analysis for the dimension verification}

The final allograft dimensions are listed in Table 1 . The variable abbreviations corresponding to the measurements used are shown in Figure 4. To verify the final section, a sensitivity analysis was performed by altering the total allograft dimensions $\left(D_{h}, D_{v}\right)$ or the orthogonal section lengths $\left(d_{h}, d_{v}\right)$. Thus, the length and angle of the diagonal section were determined by these four independent variables. In Figure 5, the dimensions were individually modified (keeping the other three variables constant according to the final dimensions in Table 1), and the section volume and surface area intactness were plotted.

For multivariable optimization, Figure 6(a) shows the horizontal and vertical allograft lengths on a two dimensional (2D) map, where the colour represents the surface intactness rate given those dimensions. For visualization purposes, the equipercentile contours were also traced (at two percent, one percent, and $0.25 \%$ increments), and the value pairs inside the dotted-blue boundary yielded surface intactness rates above $98.75 \%$ (i.e. porosity rates below $1.25 \%$ ). Similarly, Figure 6 (b) shows the horizontal section length against the vertical length and the resulting surface intactness at each value pair.

Because the slice thickness (along the superior-inferior axis) was $1.25 \mathrm{~mm}$, it was reduced to $1.25 \mathrm{~mm} / 3=0.4167 \mathrm{~mm}$ by supersampling the image using linear interpolation, yielding near-isotropic operating voxels with dimensions of $0.3906 \times 0.3906$ $\times 0.4167 \mathrm{~mm}^{3}$. The length, area, and volume values were calculated using these effective voxel dimensions. The surface intactness ratios and section volumes were calculated in the MathWorks Matlab (Natick, MA, USA) numerical computing environment, as follows.

1. For preprocessing, the image was supersampled along the Z-dimension and thresholded at 150 Hounsfield units. Gaussian smoothing was performed.

2. For each value quadruplet $\{\mathrm{dv}, \mathrm{Dv}, \mathrm{dh}, \mathrm{Dh}\}$, the following steps were performed.

2(a). The corresponding vertical, horizontal, and diagonal section slices were sampled from the segmented binary image.

2(b). Section slice areas $A_{v}, A_{h}$, and $A_{d}$ were calculated as sums of the true pixels on each slice.

2 (c). A morphological closing operation [11] was performed with a circular ( $\mathrm{r}=25$ voxels $\approx 10$ mm) structuring element for each slice.

2(d). The morphologically closed areas $A_{x, \text { closed, where } x}=\{v, h, d\}$, were calculated.

2(e). The surface intactness rate $\frac{\sum A_{x}}{\sum A_{x, \text { closed }}}$, where $x=\{v, h, d\}$, was calculated.

2(f). The section volume was calculated by sampling a heptahedron-shaped subvolume encapsulated by $\{\mathrm{dv}, \mathrm{Dv}, \mathrm{dh}, \mathrm{Dh}\}$ and outer image bounds and retrieving the sum of its true pixels.
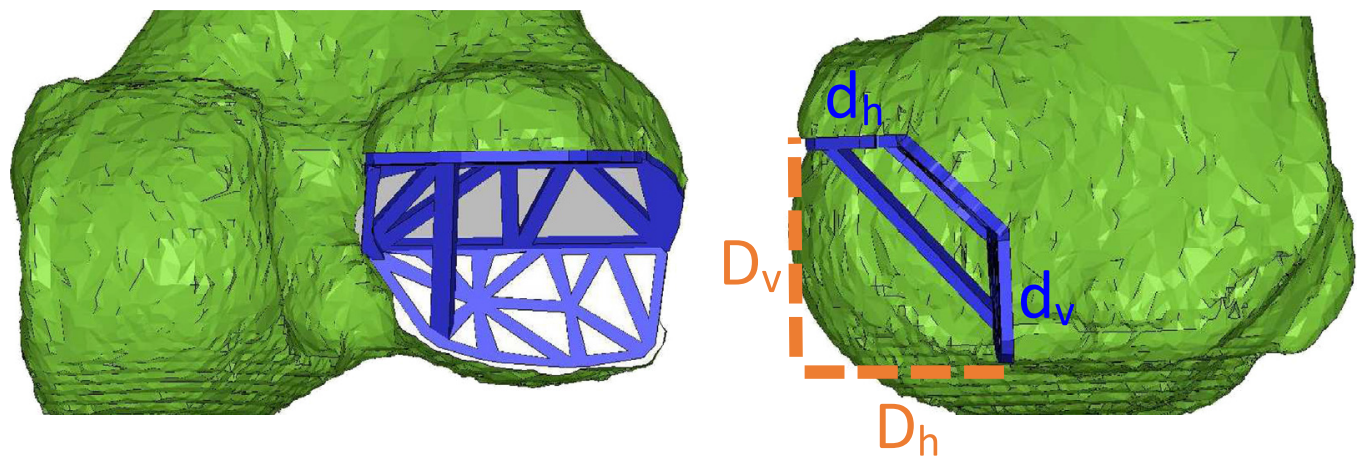

Figure 4. Small digitally designed saw templates (blue) fitted against the planned section lines, and the section measurements used in the numerical analysis. 
Table 1

Final allograft measurements.

\begin{tabular}{|c|c|c|c|c|}
\hline \multirow[t]{2}{*}{ Variable } & \multicolumn{3}{|l|}{ Value } & \multirow[t]{2}{*}{ Units } \\
\hline & Horizontal & Vertical & Diagonal & \\
\hline Total allograft dimensions $\left(D_{h}, D_{v}\right)$ & 26.56 & 29.17 & & $\mathrm{~mm}$ \\
\hline Section lengths $\left(d_{h}, d_{v}\right)$ & 11.72 & 15.42 & $20.23^{\mathrm{a}}$ & $\mathrm{mm}$ \\
\hline Section surface areas & 4.38 & 8.32 & 7.14 & $\mathrm{~cm}^{2}$ \\
\hline Section surface area, total & & 19.84 & & $\mathrm{~cm}^{2}$ \\
\hline Volume (estimate) & & 16.50 & & $\mathrm{~cm}^{3}$ \\
\hline
\end{tabular}

a Dependent variable; equal to $\sqrt{\left(D_{h}-d_{h}\right)^{2}+\left(D_{v}-d_{v}\right)^{2}}$ according to the Pythagorean theorem.

As shown in Figure 5, the chosen values coincided with the local surface intactness maxima along each respective dimension in the unidirectional sensitivity analysis. Reducing the vertical total allograft length beyond $2.5 \mathrm{~mm}$ resulted in a loss of the intactness, and for the horizontal total length, the value decreased sharply with a reduction of approximately four millimetres. Modifying the orthogonal section lengths resulted in smaller changes in the intactness; however, there were smaller changes in the total section volume, owing to the overall shape of the section.

All possible design variations could be numerically verified; therefore, a suitably defined optimal design could also be automatically dimensioned. This was the multivariate four dimensional (4D) optimization problem defined as the maximization of fitness function F: $\underset{\left\{d_{v}, D_{v}, d_{h}, D_{h}\right\}}{\arg \max } F=\frac{S I\left(d_{v}, D_{v}, d_{h}, D_{h}\right)^{\alpha}}{V\left(d_{v}, D_{v}, d_{h}, D_{h}\right)^{\beta}}$ under constraints $d_{v} \geq d_{v, \text { min }}, d_{h} \geq d_{h, \text { min }}$. The parameter SI was the surface intactness rate, the parameter $V$ was the section volume, and the $d_{\text {min }}$ values referred to the shortest section lines able to be fixated. The terms $\alpha \in \mathbb{R}$ and $\beta \in \mathbb{R}$ were weighting parameters for the fitness function. When $\alpha<; \ll \beta$, the optimization strategy emphasized
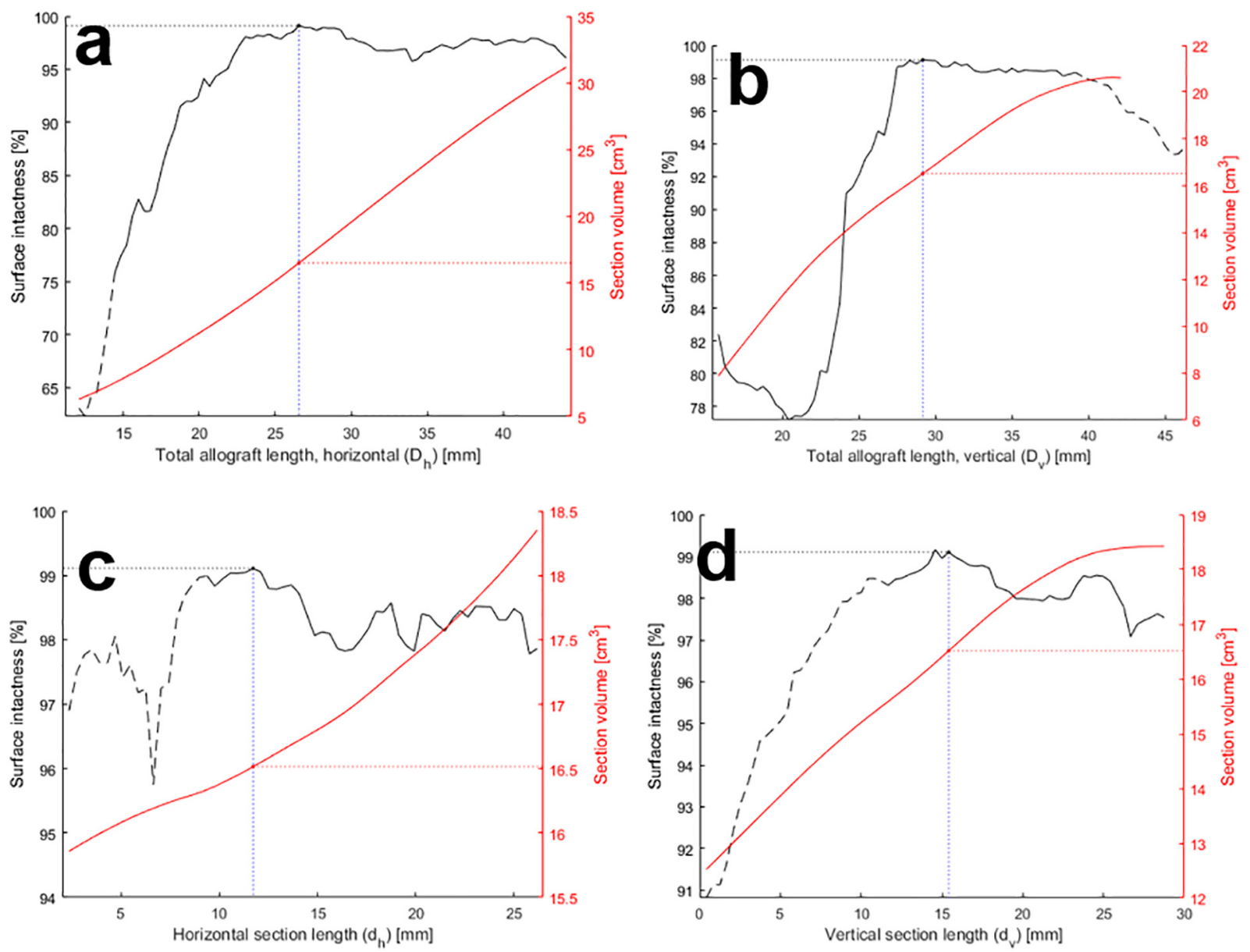

Figure 5. Surface intactness and, on the right axis, the section volume subjected to alteration of the (a) horizontal total allograft dimension $\mathrm{D}_{\mathrm{h}}$, (b) vertical total allograft dimension $D_{v},(c)$ horizontal section length $d_{h}$, and $(d)$ vertical section length $d_{v}$. The dotted lines represented the chosen value and the resulting surface intactness and section volume values. The areas with the dashed surface intactness curve denoted the ineligible dimensions. The fixation length was inadequate, or the section height (total vertical length) surpassed the height of the femoral condyle. 

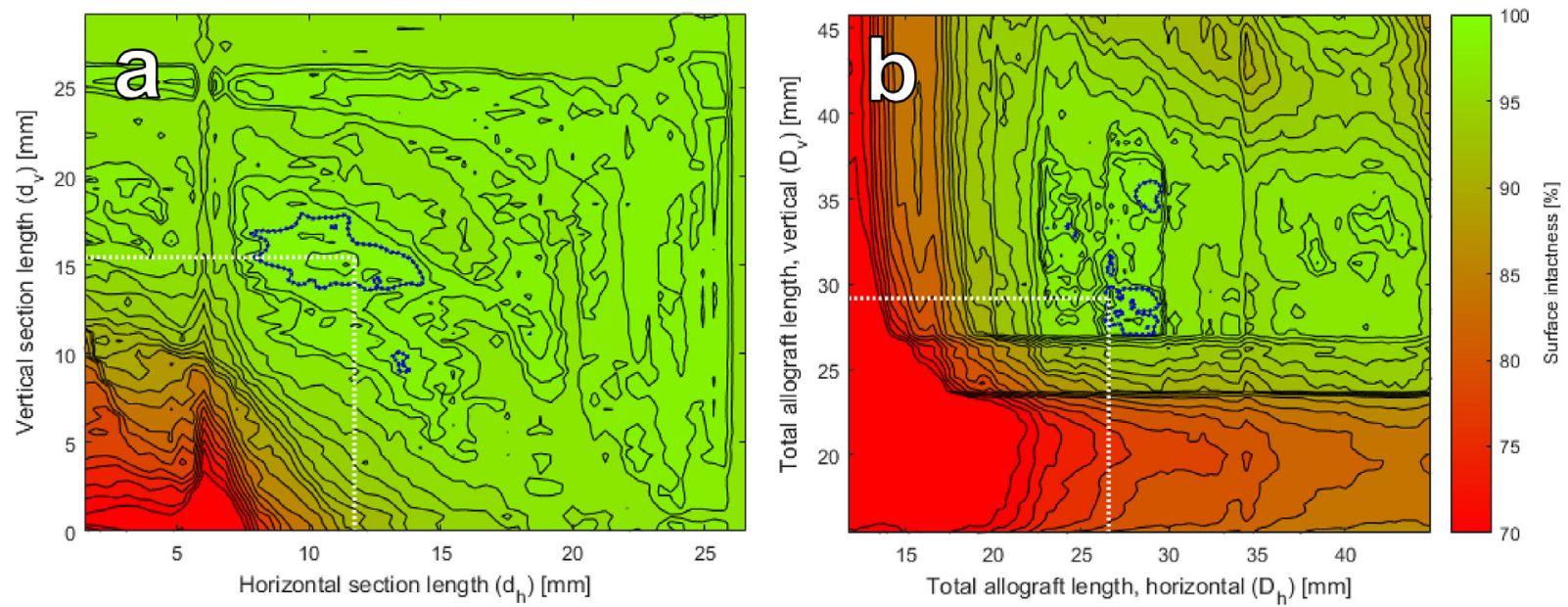

Figure 6. Surface intactness rate plotted as a multivariate result from the horizontal and vertical dimensions. (a) Alteration of the section lengths ( $d_{h}$ against $d_{v}$ ); (b) alteration of the total allograft lengths ( $D_{h}$ against $D_{v}$ ). The contours were traced at two percent intervals from $80 \%$ to $90 \%$, one percent intervals from $90 \%$ to $98 \%$, and $0.25 \%$ intervals from $98 \%$ to $99 \%$. The surface intactness rates above $98.75 \%$ were highlighted with a dotted blue contour, and the chosen dimension value pair was marked with a white dotted line.
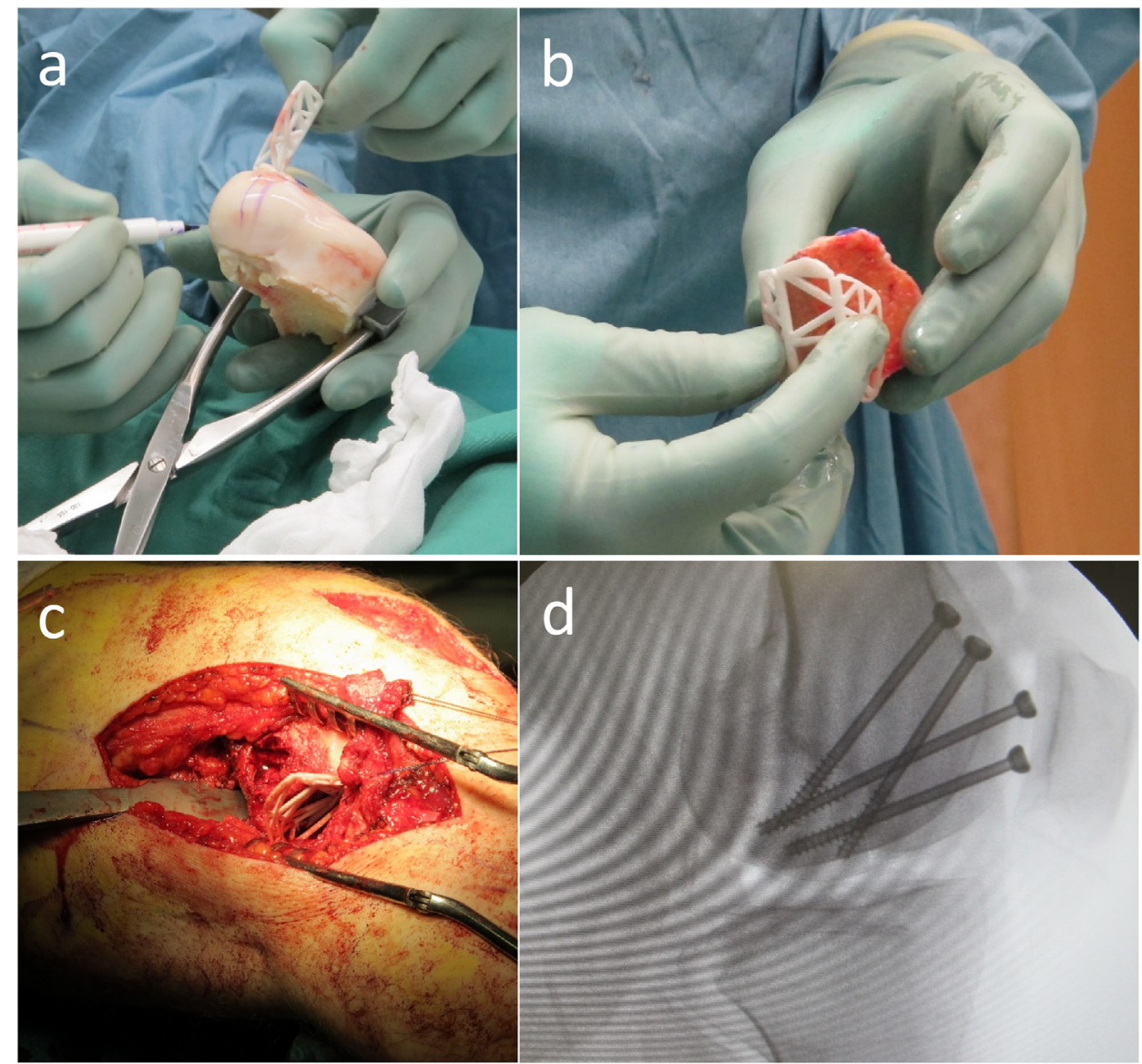

Figure 7. Surgical procedure and utilization of the saw templates. (a) Drawing of the cut lines on the cadaver bone using the larger saw template. (b) Verification of the allograft dimensions by fitting the allograft against the larger template. (c) Sectioning of the recipient knee, with section dimensions verified by fitting the smaller saw template against the bone. (d) Intraoperative C-arm image used to verify the fit of the allograft. 
volume minimization, and when $\alpha>$; $\gg \beta$, the surface intactness was maximized. Iterating over the possible variables at onevoxel ( $0.3906 \mathrm{~mm}$ horizontally, $0.4167 \mathrm{~mm}$ vertically) increments with parameters $\alpha=2, \beta=1$ yielded the globally optimal values of $\mathrm{d}_{\mathrm{v}}=13.75 \mathrm{~mm}, \mathrm{D}_{\mathrm{v}}=27.50 \mathrm{~mm}, \mathrm{~d}_{\mathrm{h}}=11.33 \mathrm{~mm}$, and $\mathrm{D}_{\mathrm{h}}=22.65 \mathrm{~mm}$. This was close to the manually chosen section line, excluding the utilized safety margin of two to three millimetres.

\subsection{Surgical procedure}

The surgical templates were sterilized using an autoclave process, and the intraoperative model was sealed in a sterile plastic bag. Then, the parts were transported to the operating room. In the surgical procedure, a lateral longitudinal approach was used. The lateral collateral ligament was detached with a bone block from the lateral femoral condyle. The popliteus tendon and lateral meniscus were left intact. The joint capsule was opened longitudinally.

The posterior inferior portion of the lateral femoral condyle was resected with the smaller sawing template (Figure 7(c)). A similar-sized osteochondral graft was cut from the fresh cadaver femoral condyle using the matching larger template (Figure 7 (a), (b)). The FOCA transplant was fixed with cannulated 4.5-mm screws in the anterior posterior direction through a short anterolateral parapatellar incision (Figure 7(d)).

The FOCA transplant fit precisely, and the articular surface of the lateral femoral condyle was congruent. The lateral collateral ligament with a bone block was reinserted with a cancellous screw and washer into the lateral femoral epicondyle. The joint capsule and soft tissue layers were closed separately. Postoperative range-of-motion and muscle exercises were initiated. During the first 12 weeks, partial weight bearing was allowed.

\section{Results}

The templates and preoperative model were designed, produced, and promptly transported to the hospital for sterilization. After receiving the DICOM images of the patient, the 3D modeling required two to three hours. The manufacture and delivery of the saw templates required $20 \mathrm{~h}$, and the preoperative models (produced in-house) required $12 \mathrm{~h}$. The 3D-printing processes required the majority of the time, as the components were produced overnight.

The digital design was shown to be viable through the CT analysis, with the numerical optimization algorithm yielding comparable design dimensions. The surgical fit of the transplanted allograft was also adequate, and during the surgical procedure, one reiteration cycle of drilling-imaging-redrilling was required. Figure 7(d) shows an intraoperative C-arm scan of the knee after the final placement and attachment of the allograft. Figure 8(a) and (b) shows postoperative X-ray radiographs, and Figure 8 (c) shows T2-weighted fast spin echo magnetic resonance imaging (MRI) images from the knee. The baseline imaging was obtained three days postoperatively and repeated at follow-up appointments.

Figure 9 shows four-month follow-up radiographs (Figure 9(a), (b)) and three-month follow-up MRI images (Figure 9(c), (d)), which show bony integration in the osteotomy line. Clinical outcome was assessed with the Knee injury and Osteoarthritis Outcome Score (KOOS) questionnaire: the results are shown in Figure 10. After the first half year of follow-up, the clinical outcome improved according to KOOS pain and symptom questionnaires. We observed degenerative changes in the allograft cartilage surface after three months in MRI images. The degenerative changes of the articular cartilage enlarged during the follow-up. Cone-beam CT examination verified that the allograft had maintained its postoperative position, and the allograft incorporated to the host bone between two and four months. Minor revascularization of the allograft was observed during follow-up with dynamic MRI imaging. However, the metal screws caused artefact and interfered in the evaluation. After eight to nine months of follow-up, the patient started having more pain and there was an obvious secondary osteoarthritis in the lateral tibiofemoral compartment.

\section{Discussion}

The ability to use digital planning, design, and manufacturing techniques offers vast potential for planning and performing complex surgical procedures. As the dimensions were derived from the CT data of the patient, spatial accuracies in the submillimetre scale were obtained. This was the first customized FOCA transplantation in the Nordic countries, where half of the weightbearing surface of the lateral femoral condyle was reconstructed with this technique. Given the novelty of this operation, the surgeons could not compare the efficiency of the surgery to prior operations. However, the digital planning and design as well as the patient-specific surgical tools and medical models enhanced the operational outcome by reducing the surgical time; therefore, the correct fit of the transplant was achievable.

The rigidity of the templates could have been improved (the PA parts were bendable when enough force was applied) by using a more constrained design. Alternatively, medical grade metals, such as titanium, could have been used instead of the chosen plastic material. However, given the tight temporal window of the operation, it could have not been procured in time. The time from medical imaging to the start of the surgical procedure was approximately $48 \mathrm{~h}$. This time included the digital design, manufacturing of the models and templates, post-processing (autoclave sterilization) of the parts, and logistics between the hospital, design and engineering partner, and manufacturer of the parts. The capability of the global metal part production is likely to increase, thus improving the metal-part lead-times and availability. Nevertheless, metal processes are slower than plastic processes, and they require support structure removal and post-processing. This increases the manufacturing time and may complicate the lean production of patient-specific titanium surgical tools to be used in time-critical surgical operations. 

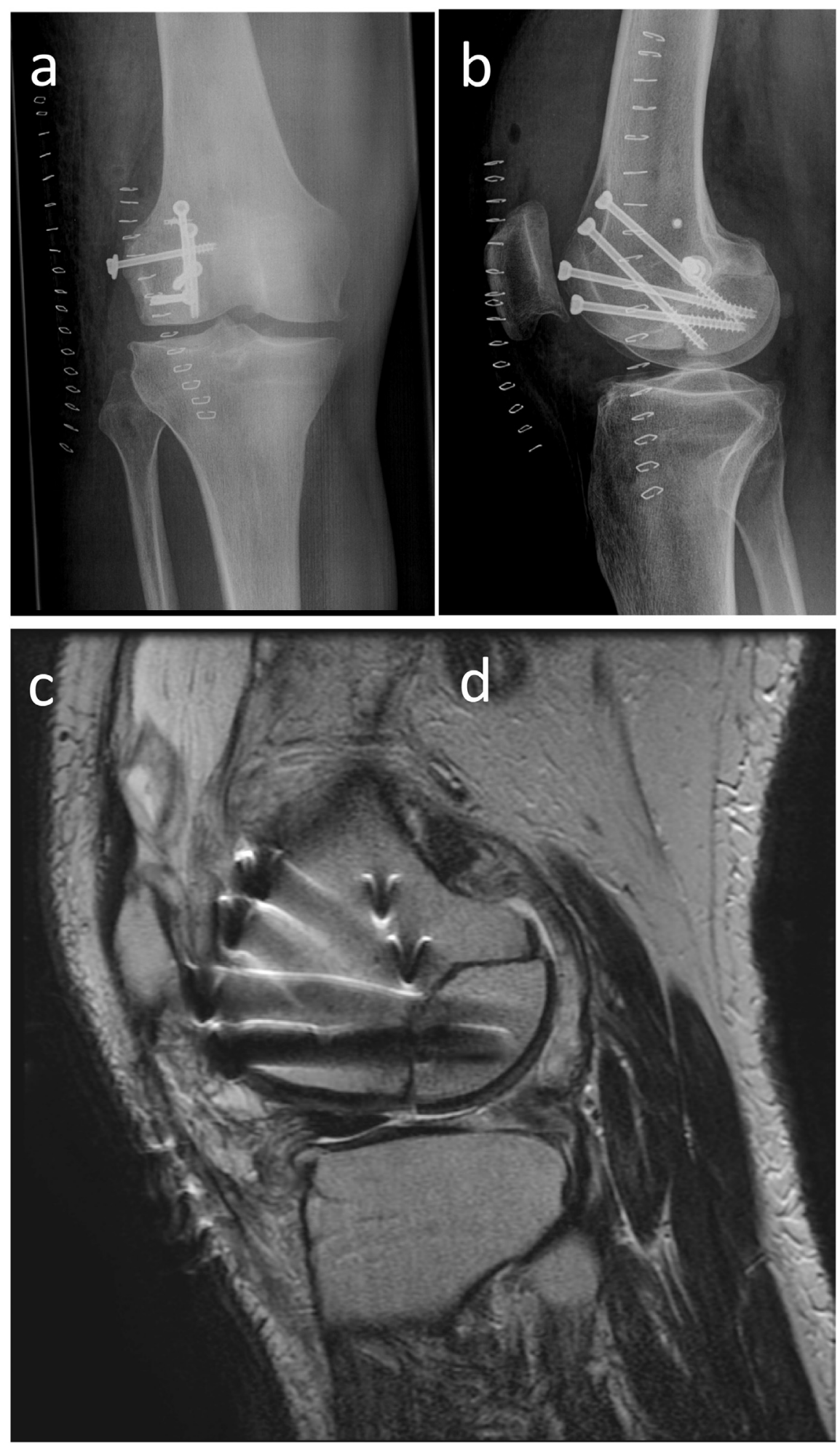

Figure 8. (a,b) Postoperative radiographs. (c) Sagittal T2-weighted fast spin echo magnetic resonance imaging. These images show good congruency of the lateral femoral condyle without any step-off in the chondral articular surface.

Instead of the 3D printing method presented, the digital operation plan could be realized with surgical navigation tools [12], or in robotic surgery. The proposed solution was more cost-effective than these methods because the digital design and calculation steps could be performed on a consumer-level laptop with a few software programs, and the manufacture of the parts could be 


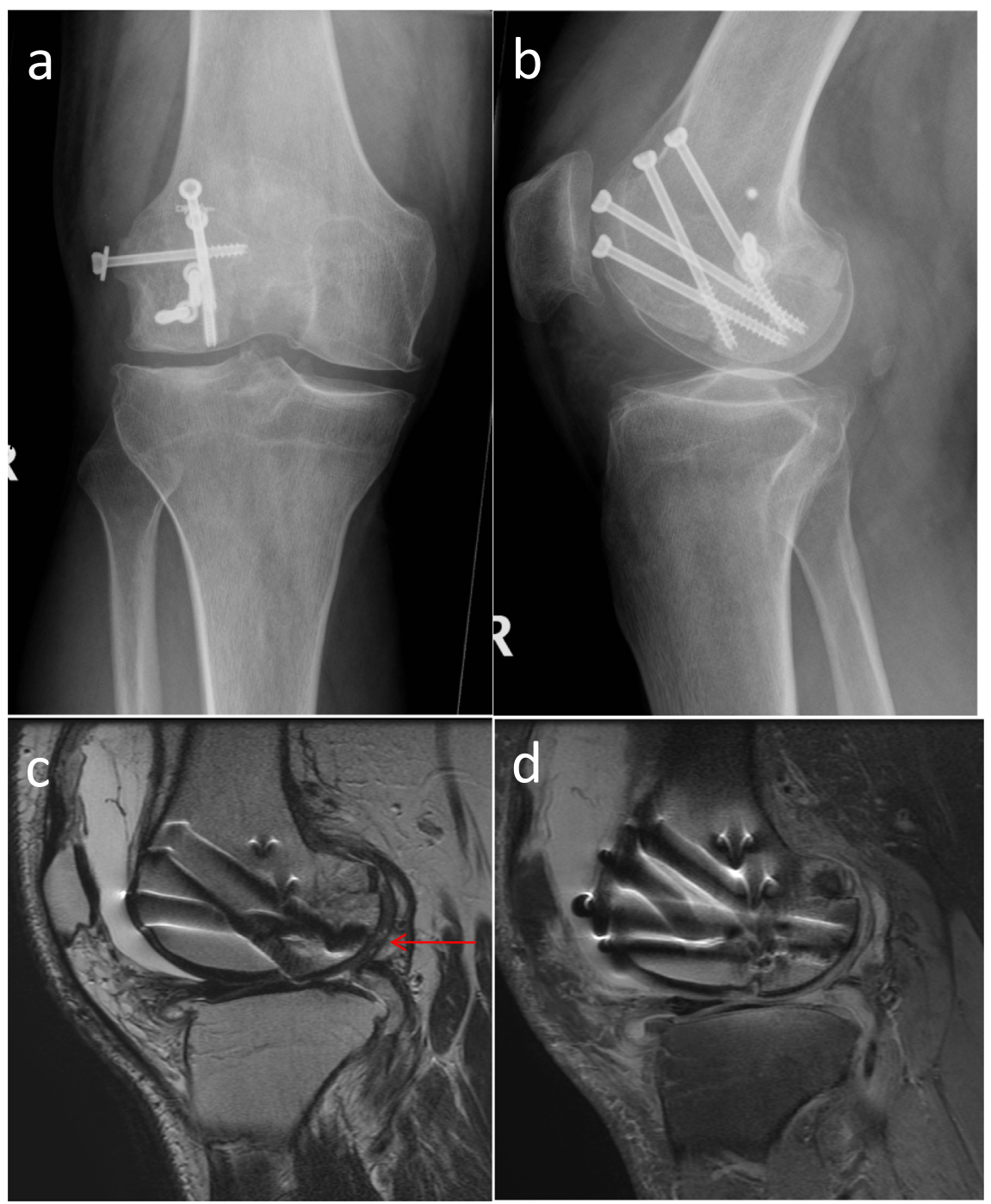

Figure 9. (a,b) Four-month follow-up radiographs show healing of the fresh cadaveric osteochondral allograft (FOCA). (c,d) Three-month follow-up MRI images show bony integration in the osteotomy line. Cartilage is well preserved, but a local cartilage defect (arrow) is seen (c).

outsourced to numerous 3D printing hubs and service bureaus. In theory, the design steps presented in this study could be automated. A possible optimization strategy was presented with a simple, customizable fitness function, and it produced results comparable to those of the manual design process. However, design automation would require considerable user input to define the boundary conditions and parameter space (e.g., the dimensions along which the part was to be optimized). No algorithm will provide these decisions out of the box. Instead, a combination of digital design, modern manufacturing methods, computational analysis or optimization tools, and surgical expertise should be used in future orthopaedic needs of the highest ambition.

\section{Conclusions}

A design method for the creation of patient-specific tools for complex autograft fitting tasks was proposed. Additionally, a numerical method for design verification and optimization was suggested, and 3D-printed surgical templates were produced and trialed within a fresh osteochondral allograft surgery on lateral condyle.

\section{Acknowledgements}

This research was partly funded by Tekes - Finnish Funding Agency for Technology and Innovation. 


\section{Clinical outcome: KOOS}

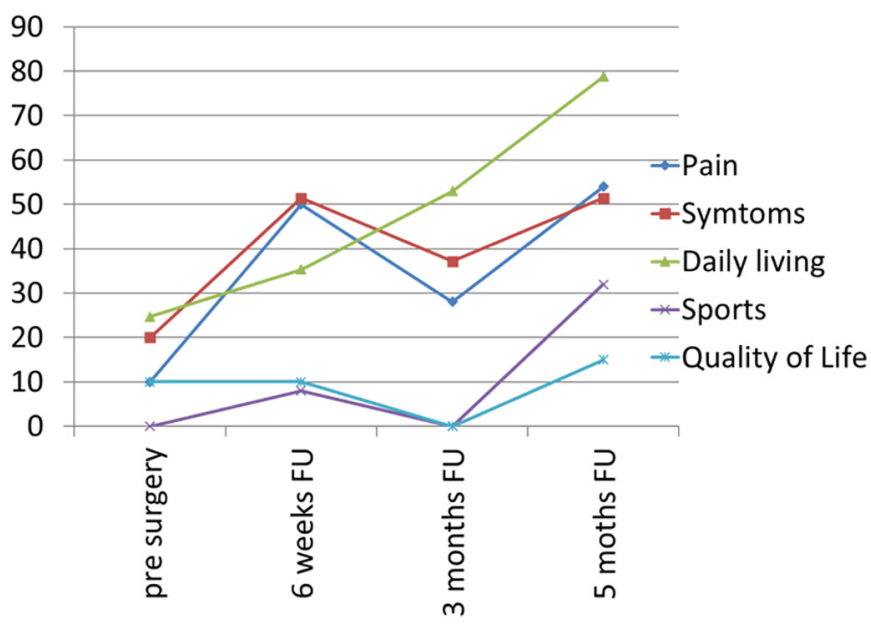

Figure 10. Clinical outcome (KOOS scores up to five months of follow-up).

\section{Declaration of Competing Interest}

The authors have no conflicts of interest related to this work.

\section{References}

[1] Tuomi J, Paloheimo KS, Vehviläinen J, Björkstrand R, Salmi M, Huotilainen E, et al. A novel classification and online platform for planning and documentation of medical applications of additive manufacturing. Surg Innov 2014;21:553-9.

[2] Atala A, Kasper FK, Mikos AG. Engineering complex tissues. Sci Transl Med 2012;4:160.

[3] Zopf DA, Nelson ME, Ohye RG, Green GE. Bioresorbable airway splint created with a three-dimensional printer. N Engl J Med 2013;368:2043-5

[4] D'Urso PS, Barker TM, Earwaker WJ, Bruce LJ, Atkinson RL, Lanigan MW, et al. Stereolithographic biomodelling in cranio-maxillofacial surgery: a prospective trial. J Craniomaxillofac Surg 1999;27:30-7.

[5] D'Urso PS, Earwaker WJ, Barker TM, Redmond MJ, Thompson RG, Effeney DJ, et al. Custom cranioplasty using stereolithography and acrylic. Br J Plast Surg 2000; 53:200-4.

[6] Erickson DM, Chance D, Schmitt S, Mathis J. An opinion survey of reported benefits from the use of stereolithographic models. J Oral Maxillofac Surg 1999;57: 1040-3.

[7] Riesenkampff E, Rietdorf U, Wolf I, Schnackenburg B, Ewert P, Huebler M, et al. The practical clinical value of three-dimensional models of complex congenitally malformed hearts. J Thorac Cardiovasc Surg 2009;138:571-80.

[8] Ngan EM, Rebeyka IM, Ross DB, Hirji M, Wolfaardt JF, Seelaus R, et al. The rapid prototyping of anatomic models in pulmonary atresia. J Thorac Cardiovasc Surg 2006;132:264-9.

[9] Huotilainen E, Paloheimo M, Salmi M, Paloheimo KS, Björkstrand R, Tuomi J, et al. Imaging requirements for medical applications of additive manufacturing. Acta Radiol 2014;55(1):78-85.

[10] Rosset A, Spadola L, Ratib O. OsiriX: an open-source software for navigating in multidimensional DICOM images. J Digit Imaging 2004;17:205-16

[11] Serra J. Image analysis and mathematical morphology. Orlando, FL: Academic Press; 1983.

[12] Gerbers JG, Van Ooijen PMA, Jutte PC. Computer-assisted surgery for allograft shaping in hemicortical resection. Acta Orthop 2013;84:224-6. 